\title{
Open inflation and the singular boundary
}

\author{
Jaume Garriga* \\ IFAE, Departament de Fisica, Universitat Autonoma de Barcelona, 08193 Bellaterra, Spain
}

(Received 6 April 1998; published 18 January 2000)

\begin{abstract}
The singularity in the Hawking-Turok model of open inflation has some appealing properties, such as the fact that its action is integrable. Also, if one thinks of the singularity as the boundary of spacetime, then the Gibbons-Hawking term is nonvanishing and finite. Here, we consider a model where the gravitational and scalar fields are coupled to a dynamical membrane. The singular instanton can then be obtained as the limit of a family of "no-boundary" solutions where both the geometry and the scalar field are regular. Using this procedure, the contribution of the singularity to the Euclidean action is just 1/3 of the Gibbons-Hawking term. Unrelated to this issue, we also point out that the singularity acts as a reflecting boundary for scalar perturbations and gravity waves. Therefore, the quantization of cosmological perturbations seems to be well posed in this background.
\end{abstract}

PACS number(s): $98.80 . \mathrm{Cq}$

Recently, Hawking and Turok [1,2] have suggested that an open universe can be created from nothing. This is an attractive possibility because it would allow one to construct open models of inflation with very simple inflationary potentials (see also [3-6]).

The new ingredient that makes their construction possible is that they allow their instanton solution to be singular. There may be some justification for this, since the Euclidean action is integrable. Moreover, if we think of the singularity as the boundary of spacetime, the Gibbons-Hawking boundary term [7] is nonvanishing and finite. This is rather coincidental, since it requires the extrinsic curvature of the boundary to increase just at the same rate as the inverse of its volume as the singularity is approached.

On the other hand, the very existence of a boundary may be considered a disturbing feature (particularly in the context of the no-boundary proposal for the wave function), and one may conjecture that the singularity is just an effective description at low energies whose structure is resolved in the framework of a better theory. Here we shall consider the possibility of regularizing the singularity with matter, so that the instanton can be obtained as the limit of a family of nonsingular geometries where the scalar field is also well behaved. As we shall see, by using this limiting procedure, the contribution of the "singularity" to the Euclidean action is different from the Gibbons-Hawking term.

The simplest way to regularize the solutions is to introduce a membrane coupled to the scalar field. The Euclidean action is given by

$$
S_{E}=\int d^{4} x \sqrt{g}\left[\frac{1}{2}(\partial \phi)^{2}+V(\phi)-\frac{\mathcal{R}}{16 \pi G}\right]+\int d^{3} \xi \sqrt{h} \mu(\phi),
$$

where

$$
\mu(\phi)=\mu_{0}-\alpha e^{\kappa \phi},
$$

*Electronic address: garriga@ifae.es and $h$ is the determinant of the metric on the world sheet of the membrane. The parameter $\mu_{0}>0$ is a positive tension which stabilizes the vacuum at $\phi=0$, and $\alpha$ is a small coupling. These parameters will not play a role once the "singular' limit is taken, but for the time being there is no harm in thinking of them as physical. The parameter $\kappa$ will be specified below. We have not written a boundary term, since our geometries will not have a boundary.

Following [1] we take an $\mathrm{O}(4)$-symmetric ansatz for the metric and the scalar field:

$$
d s^{2}=d \sigma^{2}+b^{2}(\sigma)\left(d \psi^{2}+\sin ^{2} \psi d \Omega_{2}^{2}\right) .
$$

In the absence of a membrane, the field equations for $b(\sigma)$ and $\phi(\sigma)$ are

$$
\begin{gathered}
\phi^{\prime \prime}+3 \frac{b^{\prime}}{b} \phi^{\prime}=V_{, \phi}, \\
\left(\frac{b^{\prime}}{b}\right)^{2}=\frac{8 \pi G}{3}\left[\frac{1}{2} \phi^{\prime 2}-V\right]+\frac{1}{b^{2}},
\end{gathered}
$$

where primes stand for derivatives with respect to $\sigma$.

The Hawking-Turok instanton is depicted in Fig. 1. The

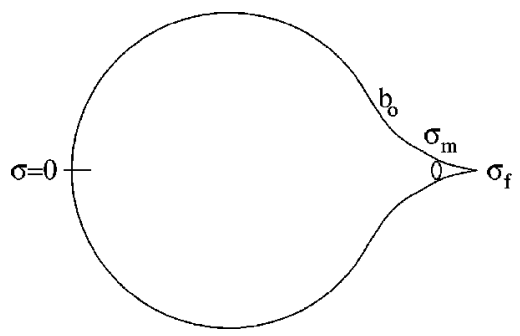

FIG. 1. Hawking-Turok singular instanton. The solution is regular at $\sigma=0$, where $b \approx \sigma$ and $\phi^{\prime}=0$. As $\sigma$ is increased, $b$ grows to a maximum value and then decreases again, reaching a second zero at some $\sigma=\sigma_{f}$, where the solution is singular. To the left of $b_{0}$ the solution is very similar to the Euclidean-de Sitter solution, but to the right it has the behavior given in Eqs. (6) and (7). The singularity can be removed by introducing a membrane coupled to the scalar field at $\sigma=\sigma_{m}$. 
solution is regular at $\sigma=0$, where $b \approx \sigma$ and $\phi^{\prime}=0$. As $\sigma$ is increased, $b$ grows to a maximum value and then decreases again, reaching a second zero at some $\sigma=\sigma_{f}$. However, this second zero is singular. Near the singularity the scale factor behaves as $[1,4]$

$$
b^{3} \approx C(12 \pi G)^{1 / 2}\left(\sigma_{f}-\sigma\right),
$$

and the scalar field as

$$
\phi \approx-(12 \pi G)^{-1 / 2} \ln \left(\sigma_{f}-\sigma\right)+\text { const. }
$$

These asymptotic expressions are valid for

$$
b \ll b_{0} \equiv C^{1 / 2} G^{1 / 4} \text {. }
$$

In spite of the singular behavior of the scalar field and the geometry, the Euclidean action is integrable.

Here, we shall take the approach of modifying the solution so that it will be everywhere regular. The idea is to surround the singularity with a spherical membrane which will act as a source for the scalar field. The interior of the membrane is replaced with a ball of (nearly) flat space. At the center of the ball, $\sigma=\sigma_{c}$, we take $\phi^{\prime}=0, b^{\prime}=-1$, and $\phi\left(\sigma_{c}\right)$ is chosen so that it matches the value of $\phi$ at the membrane. The membrane will also provide the energy momentum source necessary to match both geometries.

Substituting the $\mathrm{O}(4)$-symmetric ansatz into the Euclidean action and varying with respect to $\phi$, one easily finds matching conditions for the scalar field at the membrane. The discontinuity in the first derivative is given by

$$
\left[\phi^{\prime}\left(\sigma_{m}\right)\right]=-\alpha \kappa e^{\kappa \phi\left(\sigma_{m}\right)},
$$

where the square brackets indicate the difference between the values inside and outside, and $\sigma_{m}$ is the location of the membrane. Given that $\phi^{\prime} \approx 0$ inside the membrane and using the asymptotic form of $\phi^{\prime}$ near the external face we have

$$
\frac{C}{(12 \pi G)^{1 / 2}} \approx \alpha b^{3}\left(\sigma_{m}\right) e^{\kappa \phi\left(\sigma_{m}\right)} .
$$

The left hand side of this equation is constant. In order to obtain a nontrivial limit as $\sigma_{m} \rightarrow \sigma_{f}$ while keeping $\alpha$ finite we take

$$
\kappa \equiv(12 \pi G)^{1 / 2} \text {. }
$$

Let us now consider the back reaction of this membrane on the geometry. Einstein's equations imply the matching condition [8]

$$
\left[\frac{b^{\prime}}{b}\right]=-4 \pi G \mu(\phi)=-4 \pi G\left(\mu_{0}-\alpha e^{\kappa \phi\left(\sigma_{m}\right)}\right) .
$$

Inside the membrane, the geometry is basically flat, and we have $\left(b^{\prime} / b\right) \approx b^{-1}$. Outside the membrane, we have

$$
\frac{b^{\prime}}{b} \approx \frac{-\kappa C}{3 b^{3}} .
$$

Using Eq. (10) we find that the leading $O\left(b^{-3}\right)$ terms in Eq. (12) cancel out. The subleading terms are unimportant; they will not contribute once the size of the membrane is shrunk to zero.

Inserting the trace of Einstein's equations in Eq. (1), we find [9]

$$
S_{E}=-\int d^{4} x \sqrt{g} V(\phi)-\frac{1}{2} \int d^{3} \xi \sqrt{h} \mu(\phi) .
$$

The limit of the second term as the size of the membrane is shrunk to zero can be interpreted as the contribution of the singularity to the action of the instanton. It is given by

$$
S_{\text {sing }}=\frac{\pi^{2} C}{\kappa} .
$$

Taking into account that the trace of the extrinsic curvature of the membrane is $K=3\left(b^{\prime} / b\right)$, and using Eq. (13), we find that this contribution is actually one-third of the GibbonsHawking term $[7,4,2]$ evaluated on the external face of the membrane:

$$
S_{\text {sing }}=\frac{1}{3} S_{G H}=\frac{-1}{24 \pi G} \int d^{3} \xi \sqrt{h} K_{\text {ext }} .
$$

This conclusion is rather general. The junction condition [8] $[K]=-12 \pi G \mu$ relates the value of $\mu(\phi)$ in Eq. (14) to the jump in the trace of the extrinsic curvature. However, the jump in $K$ is dominated by the extrinsic curvature on the external face, from which Eq. (16) follows.

Note that the result in Eq. (15) does not depend on the parameters $\mu_{0}$ or $\alpha$ characterizing the membrane. The reason is that $\alpha$ has been eliminated in favor of $C$ through Eq. (10), whereas $\mu_{0}$ does not contribute in the limit $b\left(\sigma_{m}\right) \rightarrow 0$. In fact, there is no strong reason for using a coupling of the form (2). It has been chosen so that the regulator $\alpha$ remains finite as the singularity is approached. ${ }^{1}$ If we think of our membrane as a physical object, then for each $C$ and for each value of the cutoff $\sigma_{m}$, the solution only exists for specific values of $\mu_{0}$ and $\alpha$ determined by the matching conditions. One can extend this interpretation by taking the coupling $\alpha$ to be very small and allowing for a superposition of any number of membranes with positive and negative charges. In this case, the parameters $\mu_{0}$ and $\alpha$ can be thought of as continuous variables, which can be adjusted to satisfy Eq. (10) for any value of $C$ and $\sigma_{m}$.

The instability of flat space pointed out by Vilenkin [4] has an analogue in the regularized theory. Vilenkin's instanton can also be regularized with a membrane near the singularity. Just as in the cosmological case, the Euclidean world sheet of this membrane is a three-sphere. Upon analytic continuation to the Lorentzian regime, this becomes the world sheet of a spherical membrane which accelerates into the flat assymptotic region. Thus, the instability can be pictured as

\footnotetext{
${ }^{1}$ We could replace $\mu_{0}$ by $\mu_{0}+\beta e^{(\kappa / 3) \phi}$, and then $\mu_{0}$ and $\beta$ would also remain finite in the limit $b\left(\sigma_{m}\right) \rightarrow 0$.
} 
the spontaneous creation of a membrane which is a source for the scalar field. Because $\phi$ is large near the membrane, its effective energy per unit area $\mu(\phi)$ is negative. This negative energy compensates for the positive energy in the scalar field configuration, so that the total energy is zero and tunneling is allowed. In Ref. [4], a massless scalar field was considered, and there was no minimum gap to be surmounted in order for tunneling to occur (the constant $C$ could be chosen arbitrarily small). As mentioned in [4], the same seems to be true for an arbitrary potential. The regularization we suggest here can be applied for any value of $C$ and in this respect the same construction that would make the Hawking-Turok instanton acceptable also makes flat space unstable. There may be models, however, where there is a minimum height of the tunneling barrier. These models would make flat space metastable at least.

The solution of Hawking and Turok is also special with regard to the unrelated question of cosmological perturbations. In the approximation when the gravitational back reaction of the scalar field perturbations is neglected, Hawking and Turok [1] have argued that the quantization of fluctuations is marginally well defined in spite of the singularity. Indeed, after the rescaling $\phi=\chi / b$, and introducing the conformal coordinate $X=\int_{\sigma}^{\sigma_{f}} d \sigma / b(\sigma)$, the field modes obey a Schrödinger equation with a potential that behaves as $-(2 X)^{-2}$ near the singularity. This is again very coincidental, since with a stronger singularity the quantum mechanical problem would certainly be ill posed $[1,10]$.

Therefore it is important to check what happens when gravitational back reaction is included. The quantization of cosmological perturbations in $O(3,1)$-symmetric geometries (the analytic continuation of our instanton has this symmetry) was recently studied in Ref. [11]. The analysis was done in terms of the variable $\mathbf{q} \propto a \Phi / \phi^{\prime}$, where $\Phi$ is Bardeen's gauge invariant potential. The variable q obeys a Schrödinger equation with effective potential given by

$$
4 \pi G \phi^{\prime 2}+\phi^{\prime}\left(\frac{1}{\phi^{\prime}}\right)^{\prime \prime}
$$

Here, a prime indicates derivative with respect to the conformal coordinate $X$ introduced above. It is straightforward to show that the first term dominates near the singularity, behaving as $k / X^{2}$, with $k=3 / 4$. Hence, the effective potential goes to plus infinity rather than minus infinity near the singularity. Interestingly, the coefficient $k=3 / 4$ is again a critical one [10]. As mentioned above, for $k<-1 / 4$ the problem is not well posed. For $-1 / 4<k<3 / 4$ the problem is marginally well posed, since both solutions of the Schrödinger equation are square integrable near the singularity, but only one has a square integrable kinetic energy. The exception is $k=0$, for which both solutions are well behaved (this case was discussed in [5]). Finally, for $k \geqslant 3 / 4$, the basis of functions is uniquely determined by the requirement of square integrability [10], which selects one solution for each value of the energy. Thus, the singularity behaves as a reflecting boundary and the problem of quantizing the scalar perturbations seems to be well posed. In particular, this seems to preclude the possibility of scalar perturbations "streaming out" from the singularity into the universe [5]. The same comment applies to gravity waves, for which the corresponding effective potential reduces to the first term in Eq. (17) [11].

To summarize, we have shown that the contribution of the singularity to the Euclidean action depends on whether it is viewed as a true boundary of spacetime or whether it is viewed as the limit of a family of regular instantons. The second option seems preferable. The Einstein-Hilbert term may be a good approximation at low curvature, but near the singularity corrections may be expected and the finiteness of the action is hard to justify-unless the singularity is dynamically cut off in the context of a more fundamental theory. Also, we have pointed out that the quantization of linearized cosmological perturbations is well posed in the singular background. Therefore, the predicted power spectra will tend to a well-defined limit when the regulator is removed. This suggests that predictions may not be too sensitive to the details of the underlying theory, provided that the cutoff scale is sufficiently high.

It is a pleasure to thank Alex Vilenkin Takahiro Tanaka and Xavier Montes for very useful conversations.
[1] S.W. Hawking and N.G. Turok, Phys. Lett. B 425, 25 (1998).

[2] S.W. Hawking and N.G. Turok, Phys. Lett. B 432, 271 (1998).

[3] A.D. Linde, Phys. Rev. D 58, 083514 (1998); R. Bousso and A. Linde, ibid. 58, 083503 (1998); Z.C. Wu, Gen. Relativ. Gravit. 30, 1639 (1998); P. Gonzalez-Diaz, hep-th/9803178.

[4] A. Vilenkin, Phys. Rev. D 57, 7069 (1998).

[5] W. Unruh, gr-qc/9803050.

[6] J.R. Gott, Nature (London) 295, 304 (1982); J. R. Gott, in Inner Space/Outer Space, edited by E. W. Kolb et al. (The University of Chicago Press, Chicago, 1986), p. 362; B. Ratra and P.J.E. Peebles, Astrophys. J. Lett. L5, 432 (1994); Phys. Rev. D 52, 1837 (1995); M. Bucher, A.S. Goldhaber, and N. Turok, ibid. 52, 3314 (1995); 52, 5538 (1995); K. Yamamoto,
M. Sasaki, and T. Tanaka, Astrophys. J. 455, 412 (1995); A.D. Linde, Phys. Lett. B 351, 99 (1995); A.D. Linde and A. Mezhlumian, Phys. Rev. D 52, 6789 (1995).

[7] G.W. Gibbons and S.W. Hawking, Phys. Rev. D 15, 2752 (1977).

[8] W. Israel, Nuovo Cimento B 44, 1 (1966).

[9] See, e.g., J.D. Brown and C. Teitelboim, Nucl. Phys. B297, 787 (1988).

[10] See, e.g., the discussion in A. Galindo and P. Pascual, Quantum Mechanics (Springer-Verlag, Berlin, 1990). In this connection see also Ref. [5].

[11] J. Garriga, X. Montes, M. Sasaki, and T. Tanaka, Nucl. Phys. B513, 343 (1998). 